\title{
L'étrange cas de Witkiewicz et Witkacy
}

\section{Anna Saignes}

\section{OpenEdition}

\author{
Journals
}

Édition électronique

URL : http://journals.openedition.org/recherchestravaux/1271

DOI : 10.4000/recherchestravaux.1271

ISSN : 1969-6434

\section{Éditeur}

UGA Éditions/Université Grenoble Alpes

\section{Édition imprimée}

Date de publication : 15 mai 2004

Pagination : 115-128

ISBN : 2-9518254-3-9

ISSN : 0151-1874

\section{Référence électronique}

Anna Saignes, "L'étrange cas de Witkiewicz et Witkacy », Recherches \& Travaux [En ligne], 64 | 2004 mis en ligne le 20 mai 2019, consulté le 08 septembre 2020. URL : http://journals.openedition.org/ recherchestravaux/1271; DOI : https://doi.org/10.4000/recherchestravaux.1271 
Anna SAIGNES

Université Stendhal-Grenoble 3

\section{L'étrange cas de Witkiewicz et Witkacy}

Tout au long de ces travaux consacrés aux «figures paradoxales de l'auteur" n'ont cessé d'être évoqués des personnages (le faussaire, le plagiaire, le copiste...) et des pratiques (l'écriture apocryphe, le pastiche, la parodie...) dont la multiplication dans la littérature contemporaine pourrait être mise en rapport avec une crise de la légitimité de l'écrivain, voire avec le sentiment d'une difficulté liée à la représentation de l'auctoritas elle-même. A cette liste de personnages et de pratiques, qui tournent tous autour d'une idée de faux, il semble possible d'ajouter le pseudonymat : si le plagiaire signe de son nom le texte d'autrui, et le faussaire signe du nom d'autrui son propre texte, le pseudonyme, lui, signe d'un faux nom son propre texte... Un pseudonyme peut-il pour autant devenir une figure de l'auteur? Une figure paradoxale de l'auteur? Une figure paradoxale de son propre auteur?

Quelque confuses que puissent paraître ces hypothèses au premier abord, il semble bien que ce soit en ces termes qu'on pourrait tenter de rendre compte de ce qui se produit dans l'œuvre et la vie d'un des auteurs majeurs du XX' siècle polonais: Stanislaw Ignacy Witkiewicz, qui a utilisé son pseudonyme, Witkacy, de façon bien peu réglementaire et comme à rebours, semant un désordre inédit dans le discours critique qui le prend pour objet.

\section{Stanislaw Ignacy Witkiewicz}

Stanislaw Ignacy Witkiewicz (1885-1939) fut un peintre très fécond, écrivit une trentaine de pièces de théâtre et quatre romans, des essais sur la peinture et le théâtre (on a souvent rapproché sa vision de celle d'Antonin Artaud), ainsi que des milliers de pages de philosophie. Pour donner rapidement une 
idée de la place de Witkiewicz dans le paysage littéraire polonais, on pourrait dire qu'il est généralement reconnu comme une des personnalités les plus marquantes de l'entre-deux guerres et comme un de ceux qui ont engagé l'art et la culture polonais sur la voie de la modernité. Mais laissons plutôt la parole à Witold Gombrowicz qui, dans ses Souvenirs de Pologne, écrit à propos de Witkiewicz, de Bruno Schulz et de lui-même:

Nous, nous connaissions la valeur de l'originalité, non pas à l'échelle locale mais à celle de l'univers. L'objet de notre recherche, c'était l'homme en tant que tel et non pas l'homme local, l'homme polonais. Nous abordions l'art en étant formés aux techniques et aux conceptions étrangères les plus audacieuses, et prêts à tout pourvu qu'on saisisse le taureau par les cornes ${ }^{1}$.

Witkiewicz a également écrit des choses... plus difficiles à classer... Un traité sur les "narcotiques " ", où il expose ses idées sur toutes les drogues, de la nicotine au peyotl, avec compte-rendu d'expériences vécues à l'appui, et un volume où il donne ses recettes pour combattre toute sorte de petits ennuis comme les hémorroïdes et les pellicules, pour arrêter de fumer ${ }^{3}$, etc. Bref, l'œuvre de Witkiewicz est multiple et ses contours mal définis. Multiple et une à la fois pourtant, puisque parcourue par les mêmes thèmes clés, véritablement obsessionnels: l'imminence de la fin de la culture, la victoire définitive de la démocratie, qui va rendre possible le bien-être matériel de tous, mais anéantir tout ce qui a fait la valeur de l'humanité, et en premier lieu l'art. L'unité de l'œuvre witkiewiczienne n'est d'ailleurs pas exclusivement thématique: d'une œuvre à l'autre (qu'il s'agisse de fiction, d'essais ou de philosophie) on retrouve le même style, les mêmes plaisanteries, les mêmes néologismes, les mêmes jeux de mots, les mêmes procédés d'autodérision.

Pour compléter cette présentation de Witkiewicz, il reste encore à signaler la construction de ce qu'on pourrait appeler un "mythe personnel". Witkiewicz s'est rendu célèbre par ses excentricités (l'habitude de recevoir ses amis au lit le dimanche matin, nu, et de faire ensuite sa toilette en public, de mettre, au restaurant, son beefsteak dans sa poche, etc.), sa manie d'improviser à tout moment des farces dans lesquelles il fait jouer des rôles à ses amis, aux dépens d'autres gens non prévenus, son don d'imitateur... Les témoi-

I. Witold Gombrowicz, Souvenirs de Pologne, (1977), Paris, Christian Bourgois, coll. " 10/18 ", 1984, trad. Ch. Jezewski, D. Autrand, p. 168.

2. Stanislaw Ignacy Witkiewicz, Narkotyki in Narkotyki. Niemyte dusze (1932), Warszawa, PIW, 1993. En français: Les Narcotiques suivi de Les Âmes mal lavées, Lausanne, L'Âge d'homme, 1980, trad. de Gérard Conio.

3. Niemyte dusze in Narkotyki. Niemyte dusze, op. cit. 
gnages rapportent cependant que ces moments alternent avec des phases de dépression profonde. Revenons encore aux Souvenirs de Pologne où Gombrowicz décrit sa première rencontre avec notre homme:

C'est donc Bruno [Schulz], comme je viens de le dire, qui m'emmena chez Witkacy. Nous grimpons à je ne sais quel étage d'un immeuble sur cour de la rue Bracka, nous sonnons - je suis un peu troublé à cause des anecdotes qui circulent sur les extravagances et les folies de cet homme doté d'une intelligence si brillante - et soudain, dans l'entrebâillement de la porte qui vient de s'ouvrir, nous voyons apparaître un énorme nain qui se met à grandir sous nos yeux... c'est Witkacy, qui a ouvert la porte à croupetons et qui se lève lentement. Il aimait les blagues de ce genre! 4 .

\section{Witkacy}

On aura sûrement remarqué que, dans les lignes de Gombrowicz ci-dessus, il n'est pas question de Witkiewicz mais de Witkacy. En effet Witkiewicz est aussi connu (mieux connu?) comme "Witkacy», un nom qu'il a lui-même inventé. La première occurrence de ce nom se trouve dans le postscriptum d'une lettre adressée à un ami, datée d'octobre 1918, dans laquelle il demande à ce dernier de faire encadrer et exposer à Lwow des dessins et des pastels :

Mon nom en tant qu'exposant est Ignacy Witkiewicz. La signature "Witkacy" est également valable, et il se peut mểme que les choses signées ainsi soient celles auxquelles j'attache plus d'importance, à partir de $1918^{5}$.

En effet, à partir de 1918, Witkiewicz use régulièrement du nom "Witkacy». Aujourd'hui, les dictionnaires, encyclopédies, bibliographies et autres taxinomies, à l'entrée "Witkacy», indiquent qu'il s'agit là du "pseudonyme» de Stanislaw Ignacy Witkiewicz.

À y regarder de plus près cependant la notion de pseudonyme semble problématique, si l'on se penche sur l'usage que Witkiewicz fait de Witkacy. Passons sur les définitions qui donnent le pseudonyme comme «dénomination choisie par une personne pour masquer son identitém. De toute évidence, l'invention de Witkacy ne relevait pas d'un quelconque projet de dissimulation et l'équivalence Witkiewicz-Witkacy n'a jamais été un secret

4. Witold Gombrowicz, op. cit., p. 166.

5. Cité d'après Janusz Degler, "Kronika zycia i tworczosci Stanislawa Ignacego Witkiewiczan, [Chronique de la vie et de l'œuvre de S. I. Witkiewicz], in Pamietnik teatralny, I985, $\mathrm{n}^{\circ} \mathrm{I}-4$, P. 61 . 
pour personne. On pourrait en revanche retrouver à l'origine de la création du nom "Witkacy" la plupart des autres motivations qui en général soustendent le pseudonymat: rejet d'un nom mal aimé, refus œdipien du patronyme (le père de Stanislaw Ignacy Witkiewicz s'appelait Stanislaw Witkiewicz et était un critique d'art très célèbre), volonté de rompre les liens familiaux, plaisir de s'inventer un nom, amour du masque ou du travestissement, décision d'un sujet de se nommer lui-même contre les modèles ordinaires de donation des noms, désir d'un dédoublement ou d'un changement de personnalité. Toutes les hypothèses sont possibles. Le problème tient plutôt à l'usage: en effet, le pseudonyme, quelle que soit sa motivation, sert généralement à opérer un découpage de la personne. Bernard Offner définit par exemple le pseudonyme comme nom "créé par celui qui le porte" et ne désignant pas "toute la personne" mais "seulement un aspect volontairement distinct de celle-ci : l'écrivain, l'artiste, l'acteur, le combattant, le ban$\mathrm{dit}^{6}$ ", en remarquant qu'il se peut aussi que le pseudonyme envahisse toute la vie de celui dont il désignait à l'origine un aspect, et finisse par devenir le nom légal. Dans le cas Witkiewicz et Witkacy, il est impossible de circonscrire l'" aspect " de la personne que désigne "Witkacy".

En effet Witkiewicz a signé de son vrai nom, Stanislaw Ignacy Witkiewicz, toutes ses œuvres romanesques, dramatiques ou philosophiques, en d'autres termes... tout ce qu'il a écrit... Witkiewicz semble donc prendre le contre-pied de la plus ancienne définition du pseudonyme ${ }^{7}$, celle qui propose "qui écrit sous un faux nom ", puisqu'il n'a jamais rien écrit sous un faux nom! La signature "Witkacy" ne figure qu'au bas de tableaux... Witkiewicz serait l'écrivain et Witkacy le peintre? Hélas, certains portraits sont signés Witkiewicz... Il s'agit cependant de portraits maintenus dans un style réaliste, contrairement aux compositions délirantes peuplées de créatures monstrueuses qui, elles, sont signées Witkacy... Voilà qui pourrait suggérer une ligne de partage un tant soit peu satisfaisante si... Witkiewicz ne signait pas "Witkacy» les lettres qu'il écrit à ses amis... Dans la correspondance Witkacy se décline d'ailleurs d'une infinité de manières plus loufoques les unes que les autres. On y trouve ainsi :

- des formes qui correspondent aux diminutifs de Witkacy selon les modèles de formation des diminutifs en polonais: Witkas, Witkacek des variantes francisées: Saint Witkacy à la Fourchette, de Saint Vitecasse, Witkatze

6. Bernard Offner, Pour un dictionnaire des pseudonymes dans les lettres françaises du XX siecle, Paris, Bernard Offner, 1999, p. 69.

7. Voir Maurice Laugaa, La Pensée du pseudonyme, Paris, PUF, 1986, p. 37. 
- des variantes latines, formées à la manière des noms latinisés de la Renaissance : orthographiés à la polonaise: Witkrejus, Witkacjusz ou à la latine: Vitkatius, Vitkacius

- une variante hindoue: Mahatma Witkac

- une variante qui évoque vaguement l'accent des Tatras: Witkos

- une variante prussienne: Crof von Witkaczy.

Et les choses se compliquent encore si l'on remarque que Witkacy est un personnage des fictions de Witkiewicz ou encore un personnage fictif apparaissant dans des écrits qui ne relèvent pas de la fiction.

\section{Witkacy, personnage fictif et personnage de fiction}

Un inventaire exhaustif et systématique des occurrences de Witkacy et de ses variantes reste à faire. Il semble néanmoins possible d'affirmer que le phénomènè se met en place au début des années 20 , c'est-à-dire peu de temps après la création du faux nom. Il est par exemple déjà présent dans un article de I92I, intitulé "Du dandysme à Zakopane ${ }^{8}$ ", écrit sur commande pour une revue locale et qui entreprend de tourner en dérision les artistes et intellectuels "branchés" qui peuplent Zakopane. Witkiewicz y accumule des citations: on en trouve d'abord une en français, qu'une note de bas de page (de l'auteur) attribue (à juste titre) à Malesherbes ("C'est le privilège d'un vrai géniè et surtout d'un génie, qui ouvre une carrière, de faire impunément de grandes fautes"), suit la devise de l'Ordre de la Jarretière travestie ("Honni soit qui pense que je suis un mégalomane $川$ ), puis une autre en anglais ("There is no beauty without some strangeness in it's proportions"), attribuée en note (par l'auteur et là encore à juste titre) à Bacon. Enfin, entre guillemets, on peut lire la maxime suivante: "les cercueils avantagent les hommes, tout comme les cadres avantagent les tableaux". Celle-ci est attribuée en note à... Witkacy. A la fin de l'article, toujours en note, l'auteur nous recommande encore vivement de lire l'Allgemeine Gespenstertheorie de Crof von Witkaczy. L'article est signé "Genezyp Kapen", un faux nom, un pseudonyme, que Witkiewicz a inventé pour signer son premier roman, écrit vers 1909 et jamais publié.

8. S. I. Witkiewicz, "O Dandyzmie zakopianskim", in Bez kompromisu. Pisma krytyczne i publicystyczne [Sans compromis. Ecrits critiques et articles], Warszawa, PIW, I976, p. 503. Zakopane est une petite station des Tatras, promue, au tournant des $\mathrm{XIX}^{*}$ et $\mathrm{XX}^{*}$ siècles, capitale culturelle et artistique de la Pologne. La famille Witkiewicz s'y installe au début des années 1890. 
Avec le troisième roman de Witkiewicz, L'Inassouvissement ${ }^{9}$, Witkacy entre dans le monde de la fiction. On apprend, à l'occasion d'une sortie au théâtre (il s'agit d'un théâtre expérimental très en vue) envisagée par des personnages du roman, que "quelqu'un de très suspect, qui se fait passer pour le petit-fils légitime de Witkacy, ce morveux de Zakopane", y joue de petits rôles $^{\mathrm{T}}{ }^{\circ}$. Un peu plus loin, un des personnages s'assoit sur un "pouf orange Witkacy ".. On retrouve ce procédé dans Les Cordonniers, dernière pièce de Witkiewicz, achevée en 1934. Un des personnages y prononce la réplique suivante: "Witkacy, ce morveux de Zakopane, a essayé de me convaincre d'étudier la philosophie, mais même ça, je n'en ai pas été capable ${ }^{12}$ ». Enfin, dans le dernier roman, inachevé (ou plus exactement dont la seconde partie a été perdue), L'Unique Issue ${ }^{13}$, le phénomène est récurrent. Dès le début, Izydor, le personnage central qui a pour ambition de construire un système philosophique, se donne comme modèles deux esprits particulièrement rigoureux, Spinoza et... Witkacy:

Il fallait en finir avec ce fouillis et remplacer l'écheveau de pensées en lambeaux par une suite logique et compréhensible pour tous de concepts et de propositions s'enchainant les uns aux autres, quelque chose dans le style de l'Éthique de Spinoza ou du système de l'autre morveux de Zakopane, Witkacy de Krupowa Rownia ${ }^{14}$.

Tout au long du roman il sera encore souvent question de Witkacy, inventeur de mots dénués de sens ${ }^{15}$, auteur d'une valse ${ }^{16}$, auteur d'une classification des drogues ${ }^{17}$, etc. Il arrive aussi qu'on cite des opinions émises à son sujet par d'autres personnes, réelles ou imaginaires. En contemplant son propre tableau, un des personnages du roman se dit:

9. S. I. Witkiewicz, Nienasycenie, Warszawa, PIW, 1992 (1930). En français: L'Inassouvissement, Lausanne, L'Âge d'Homme, I970, trad. d'Alain Van Crugten. Il est intéressant de remarquer que le personnage central de ce roman s'appelle Genezyp Kapen, comme l'auteur présumé de l'article dont nous venons de parler et le signataire du premier roman.

Io. Ibid., p.389. Toutes les traductions des citations de Wirkiewicz sont de nous.

II. Ibid., p. 440, en français dans le texte.

12. S. I. Witkiewicz, Szewcy, in Dramaty (1934), Warszawa, PIW, 1962, p. 5IL. En français: Les Cordonniers, Paris, Gallimard, 1969, trad. de Koukou Hanska et Jacques Lacarrière.

13. S. I. Witkiewicz, Jedyne Wyjscie, Warszawa, PIW, 1993 (1968, achevé en 1933). En français: L'Unique Issue, Lausanne, L'Âge d'Homme, 2001, trad. de Gérard Conio.

I4. Ibid., p. 18. Krupowa Rownia est une rue de Zakopane.

I5. Ibid., p. 148.

16. Ibid., p. I56.

17. Ibid., p. 198/199. 
"Des explosions de cocaïne, qui au fond ne sont pas des explosions" comme a écrit, à tort d'ailleurs, à propos de Witkacy, ce morveux de Zakopane, un certain personnage de classe inférieure, ne comprenant pas qu'il commettait une cochonnerie cardinale ${ }^{18}$.

Witkacy est évoqué et convoqué à tout bout de champ. Rien d'étonnant à ce qu'il se mette à vivre sa propre vie, indépendante de celle de l'auteur de L'Unique Issue; c'est ce que nous pouvons constater lorsqu'un des personnages du roman, déplorant la faiblesse du niveau intellectuel de ses contemporains (sujet cher à Witkiewicz soit dit en passant), remarque :

Heureusement qu'au moins Chwistek a été assassiné par des sbires à la solde de Witkacy, mais aujourd'hui les gens de cette sorte proliferent comme la logique elle-même ${ }^{19}$.

Une note de bas de page précise:

C'est ce que disait hier l'élite des cancaniers municipaux dans les pissotières du dancing "Excelsior" - demain la lie et les bas-fonds du monde littéraire en parleront dans les boîtes de nuit des faubourgs. Ce n'est ni drôle, ni spirituel, mais seulement très vrai ${ }^{20}$.

\section{Faisons le point...}

Ce bref (et non exhaustif) parcours des usages de "Witkacy" semble suffisant pour montrer à quel point il est difficile de tracer une ligne de démarcation nette et stable entre Witkiewicz et Witkacy, et entre réalité et fiction. Witkacy est à la fois un personnage de fiction et celui qui signe les lettres que Witkiewicz écrit à ses amis, mais aussi le peintre; Witkiewicz ne signe pas les lettres mais signe les romans, les pièces de théâtre et les écrits philosophiques, et ne signe pas les tableaux (en règle générale). Enfin, lorsqu'on lit les témoignages des contemporains de Witkiewicz-Witkacy, on trouve, indifféremment Witkiewicz et Witkacy. Par exemple Czeslawa Okninska, qui a été sa dernière compagne, et qui a survécu à une tentative commune de suicide en 1939 (à laquelle Witkiewicz, lui, n'a pas survécu), dans ses témoignages parle toujours de Witkacy ${ }^{2 I}$. Bref, de toute évidence, il est absolument impossible

\section{Ibid., p. 138.}

19. Ibid., p. 217. Leon Chwistek (1884-1944) est un des principaux représentants du néopositivisme en Pologne. Le néopositivisme, dans la mesure où il élimine la métaphysique du champ de la philosophie, est sans cesse décrié par Witkiewicz.

20. Ibid.

2I. Le récit de Czeslawa Okninska est intégralement reproduit dans: Janusz Degler, "Kronika zycia i tworczosci Stanislawa Ignacego Witkiewicza", op. cit., p. I34 sqq. 
d'affirmer que Witkiewicz est l'homme et Witkacy l'artiste (peintre et écrivain). Et la question se complique encore lorsqu'on passe à la fiction. Witkacy s'y voit attribuer des œuvres réelles du vrai Witkiewicz, mais aussi des ceuvres, des actes et des idées inventés de toutes pièces (comme l'Allgemeine Gespenstertheorie citée ci-dessus par exemple).

Ce flottement de la nomination est confirmé par les usages en vigueur dans le discours critique (ou plus généralement dans tout discours) sur Witkiewicz. Les deux noms, Witkiewicz et Witkacy, s'y côtoient naturellement et cohabitent pacifiquement. Pour la majorité des critiques il est toujours question de Witkacy, d'autres ne parlent que de Witkiewicz, une troisième catégorie (plus réduite peut-être) emploie tantôt Witkiewicz, tantôt Witkacy. Certes, quelques lignes de partage ont parfois été suggérées. La plus répandue voit en Witkacy le «double mauvais». Elle est étayée par la récurrence du thème du double dans l'œuvre de Witkiewicz: en effet, son théâtre fourmille de doubles, ses portraits ou autoportraits fonctionnent souvent par paires. Il y a par exemple ces deux autoportraits (autoportrait se dit chez Witkiewicz: "auto-Witkacy») de 1938, exécutés dans un format et des tons identiques, dont l'un est intitulé $\mathrm{Dr}$ Jeckyll et l'autre Mr Hyde. WitkiewiczDr Jekyll et Witkacy-Mr Hyde? Dans l'étrange cas Witkiewicz et Witkacy, il s'agirait non pas tant d'une opposition entre le bien et le mal, que d'une tension entre la dimension tragique de la vision witkiewiczienne d'une part et la forme grotesque dans laquelle elle s'exprime de l'autre. En d'autres termes Witkiewicz serait l'artiste, Witkacy le bouffon. Witkiewicz serait le peintre et l'écrivain, Witkacy, le clown provocateur, malappris, qui reçoit ses amis au lit, fait sa toilette en public, organise des farces, provoque des scandales. Witkiewicz aurait écrit des pièces de théâtre, des romans, et des essais, et Witkacy nous aurait laissé des récits d'expériences psychédéliques, des conseils pour arrêter l'alcool et le tabac, et des recettes de remèdes contre les pellicules. Une des premières études consacrées à Witkiewicz, en 1958 (Witkiewicz avait été totalement "oublié" jusqu'en 1956), s'intitule "Les trois légendes du dénommé Witkacy ${ }^{22}$ ": l'auteur y déplore la «légende biographique " qui a fait que l'œuvre de Witkiewicz a été mal comprise, les bouffonneries de Witkacy ayant détourné l'attention du - et empêché de prendre au sérieux le-caractère tragique et prophétique de la vision catastrophiste de Witkiewicz.

Moins simpliste semble l'hypothèse de la mise à distance de ses propres idées, de soi, à une étape antérieure de son itinéraire d'artiste, d'homme de

22. Kazimierz Wyka, "Trzy legendy tzw. Witkacego", Tworczosc, 1958, n 10, p. 122-135. 
lettres et de philosophe. En effet Witkiewicz, dans sa première "vraie" œuvre, intitulée Les formes nouvelles en peinture et les malentendus qui en découlent, a élaboré un système esthétique, "la théorie de la Forme Pure" (avec des majuscules certes un peu emphatiques), selon laquelle, en simplifiant beaucoup, dans l'œuvre d'art seule la forme (composition de couleurs et de formes) compte et non le sujet. Witkiewicz semble ensuite avoir eu des difficultés pour mettre en œuvre son programme et a refusé d'en tirer toutes les conséquences: en peinture il n'est jamais passé à l'abstraction, ses pièces de théâtre ne sont pas dénuées de sens, et ses romans encore moins. Witkiewicz se serait ainsi rendu compte qu'il ne pouvait réaliser son programme, que sa théorie était erronée. Si l'on pose que Witkacy est le théoricien de la Forme Pure (le pseudonyme a été effectivement forgé à peu de choses près au moment où le traité d'esthétique a été écrit), le peintre débutant qui y croit encore, Witkiewicz pourrait être celui qui a compris ses erreurs " de jeunesse» et fait de Witkacy, de ce théoricien et peintre, un bouffon. Le grotesque résulterait de la tension entre la théorie et la pratique, de l'incompatibilité entre l'idéal et la réalité.

Toutefois dans la répartition des signatures entre "Witkiewicz" et "Witkacy", dont nous avons donné un aperçu ci-dessus, rien ne confirme ces hypothèses. Witkiewicz et Witkacy fonctionnent avant tout comme deux hypothèses de lecture, deux figures de l'auteur concurrentes qui limitent, chacune à sa manière, la prolifération du sens. Bouffonnerie d'un côté, tragique de l'autre. Dans les faits, l'usage des noms reste aléatoire et flottant: des monographies sur l'œuvre romanesque ou dramatique de Witkiewicz s'intitulent "L'œuvre romanesque de Witkacy", ou "Le thêtre de Witkacy " alors que, rappelons-le, Witkacy n'a signé aucun roman ni aucune pièce de théâtre. Les usagers s'obstinent à considérer comme contingent le flottement entre les deux noms.

La question de la relation entre Witkacy et Witkiewicz se heurte donc en dernière instance à l'explication par le jeu. Maurice Laugaa affirme dans $\mathrm{La}$ Pensée du pseudonyme ${ }^{23}$ que les définitions modernes du pseudonyme éliminent l'idée du faux et du mensonge pour laisser la place à celle d'une liberté du nom. L'État et la société modernes reconnaîtraient ainsi au sujet le droit de choisir son nom, mais lui dénieraient corrélativement le droit au faux et au vrai, et par conséquent remettraient en cause sa place dans une économie du monde. Le pseudonyme entrerait ainsi dans le domaine du non-sérieux, oscillant entre l'amusement, l'insignifiance et la bizarrerie. L'usage "à

23. Maurice Laugaa, op. cit., p. 76 . 
rebours" du pseudonyme par Witkiewicz pourrait constituer une variante extrême de ce phénomène. On peut rappeler ici le goût du masque et du déguisement dont témoignent la peinture et les photographies de Witkiewicz: sur ses autoportraits, ou ses "auto-witkacy" comme il les appelle lui-même, il se figure volontiers pilote d'avion, businessman, prêtre, ouvrier. "Witkacy" ne serait qu'un déguisement parmi d'autres, fréquemment revêtu, pour se livrer avec le public à des parties de cache-cache dont personne n'ignore le caractère exclusivement ludique. Bref, il s'agirait de brouiller les pistes pour le plaisir de les brouiller.

Mais voilà que le public s'est pris au jeu! Witkiewicz est devenu Witkacy. Il est sans doute curieux que toutes les tentatives d'analyse de l'étrange cas de Witkiewicz et Witkacy s'enferment dans un système binaire, qui met face à face Witkacy et Witkiewicz et exclut une troisième instance dont la part est tout aussi importante: le lecteur, qui est l'usager du nom. C'est bien ce lecteur qui décide d'appeler l'auteur de L'Inassouvissement ou des Cordonniers Witkiewicz ou Witkacy. Maurice Laugaa rappelle que : "Si l'un des traits pertinents qualifiant le pseudonyme tient à la décision d'un sujet de se nommer lui-même, contre les modèles ordinaires de donation des noms, il reste à explorer, dans cette décision et dans ses conséquences la part et l'emprise de l'Autre " ${ }^{24}$. En enfermant dans la sphère de l'amusement, de l'insignifiant et du bizarre, l'utilisation du pseudonyme par Witkiewicz, le discours critique non seulement dénie à Witkiewicz un droit au vrai et au faux mais encore dissimule (se dissimule?) son propre rôle dans le processus. Il semble donc que pour répondre à la question "qui est Witkacy?", il faut dépasser l'idée d'une simple opposition Witkiewicz-Witkacy et envisager la question sous la forme d'une interaction entre Witkiewicz, Witkacy et les récepteurs du nom.

\section{Witkacy ou le double fantasmé}

Revenons aux œuvres de fiction, où les mentions de Witkacy s'affirment et se multiplient à partir de la fin des années 1920. Souvenons-nous d'abord que les romans de Witkiewicz se déroulent dans le futur (on parle de fictions socio-historiques, ou politiques). En l'absence de repères chronologiques, on peut évaluer le temps de l'action grâce à des allusions à des personnages réels, dont on apprend qu'ils sont devenus très vieux ou dont on évoque la postérité. Il s'agirait donc successivement, pour les trois romans, du milieu du

24. Maurice Laugaa, op. cit., p. 7. 
$\mathrm{XX}^{c}$ siècle, de la fin $\mathrm{du} \mathrm{XX}^{c}$, et du début du $\mathrm{XXI}^{e}$. A l'époque où se déroule l'action des romans Witkiewicz est donc probablement mort, ou du moins très âgé; il appartient au patrimoine artistique et littéraire du passé. Et en effet lorsque Witkacy apparaît dans les œuvres de fiction de Witkiewicz, ce n'est jamais "personnellement", il n'intervient pas directement dans la fiction; c'est toujours comme quelqu'un de connu, une personnalité du "monde des arts et des lettres", quelqu'un qu'on donne en exemple, qu'on cite, voire sur lequel on cite des opinions ou des phrases prononcés ou écrites (réelles ou imaginaires?) par d'autres. Lorsqu'il est question de Witkacy, il y a presque toujours une superposition de voix, (quelqu'un cite quelqu'un qui a dit que...) qui contribue à faire de Witkacy un personnage public, universellement connu, voire mythique. On le met sur le même plan que Malesherbes, Bacon ou Spinoza. On parle de l' " orange Witkacy" comme du "vert Véronèse". Il devient la victime de rumeurs, grand-père, auteur d'œuvres que Witkiewicz n'a jamais écrites (tout en se voyant attribuer des œuvres effectivement écrites par Witkiewicz, comme nous l'avons vu).

L'on pourrait alors proposer l'hypothèse que Witkacy est (ou du moins devient avec le temps, mais assez rapidement) le reflet de Witkiewicz dans le regard déformant de l'Autre, que sous les traits de Witkacy, Witkiewicz se projette dans le regard de la postérité (ou de ses contemporains), et se fantasme dans les fantasmes de l'Autre. Non plus en prêtre ou en pilote d'avion, comme dans ses autoportraits, mais en écrivain, philosophe et peintre. Reprenons un des exemples déjà cités:

Il fallait en finir avec ce fouillis et remplacer l'écheveau de pensées en lambeaux par une suite logique et compréhensible pour tous de concepts et de propositions s'enchaînant les uns aux autres, quelque chose dans le style de l'Éthique de Spinoza ou du système de l'autre morveux de Zakopane, Witkacy de Krupowa Rownia ${ }^{25}$.

La périphrase "l'autre morveux de Zakopane", ainsi que la mention de Zakopane et de Krupowa Rownia, paradigmes de la province et de la périphérie, à côté du nom de Spinoza, produisent sans nul doute un contraste comique. N'y a-t-il vraiment là qu'autodérision? A la fin de la phrase le lecteur est renvoyé à une note où il peut lire: "J'écris ainsi pour créer un lien de sympathie entre moi-même et le lecteur, nourri de Wiadomosci Literackie ${ }^{26}$ ". De toute évidence, il y a dans ces phrases une superposition de voix: d'une part celle d'Izydor, philosophe du XXI' siècle et figure centrale du roman, qui

25. Jedyne wyjscie, op. cit., p. 18.

26. Wiadomosci literackie [Les Nouvelles littéraires] est la principale revue littéraire de l'entre-deux-guerres polonais. 
reconnaît Witkacy comme modèle de rigueur au même titre que Spinoza, et de l'autre une formule " toute faite", et d'ailleurs récurrente, «le morveux de Zakopane", qu'Izydor emprunte au discours "en vigueur" sur Witkiewicz, où, plus précisément, au discours sur Witkiewicz, tel que Witkiewicz le perçoit ou veut le percevoir, car la périphrase relève bien évidemment d'un niveau de langue qui n'est pas celui de la revue. Witkacy apparaît bien comme une entité protéiforme, susceptible de se modeler sur une variété de figures de l'auteur.

Force est de constater que Witkiewicz se sert souvent de Witkacy pour régler ses comptes en même temps que pour rectifier l'image qu'il laissera à la postérité. Ainsi, lorsqu'un des personnages de L'Unique Issue se rappelle, en contemplant son propre tableau, une phrase écrite sur Witkacy (exemple vu plus haut) et qui suggère que celui-ci peint sous l'effet de la cocaïne, une note de bas de page ajoute: "Je précise une fois de plus, que je n'ai jamais été dépendant à la cocaïne ni même à l'alcool, seulement au tabac. Et même ça, je m'en suis débarrassé. Lisez, bon Dieu, mes Narcotiques!27 „. On aurait pourtant sans doute tort de prendre Witkiewicz au pied de la lettre car s'il semble vouloir rectifier une image de lui-même comme cocaïnomane (c'està-dire, plus généralement, comme artiste décadent) en note, dans le corps du texte il contribue bien à la perpétuer. Witkiewicz n'a cessé de lutter contre ce mythe qu'il alimente lui-même, qu'il construit tout en le déconstruisant, qu'il déconstruit tout en construisant. Les deux processus sont inséparables. Ces lignes de la préface de L'Inassouvissement illustrent parfaitement le phénomène:

Un jour, alors que j'ai achevé son portrait une dame me dit: "J'avais tellement peur de vous, je me demandais : comment je vais tenir une heure avec un homme aussi terrible - et au fond vous êtes tout à fait normal et même bien élevéw. Les mères ont peur de commander chez moi les portraits de leurs filles et même les hommes adultes viennent avec des mines pas rassurées, comme s'ils s'attendaient à ce que, au lieu de dessiner, je me mette à leur arracher les dents ou crever les yeux avec un crayon... ${ }^{28}$

Le double jeu est évident: rectification d'une image soi-disant déformée mais en même temps perpétuation du mythe, sous couvert de la caricature. Il ne fait nul doute que l'effet de surprise est source de jouissance. Sans mythe, pas de surprise, donc pas de jouissance.

Witkacy n'est ni le double bouffon de Witkiewicz, ni Witkiewicz-jeunethéoricien de la Forme Pure caricaturé par Witkiewicz-mûr, ni même un 
mythe qui renvoie le lecteur à ses propres fantasmes d'écrivain-dandy, décadent, vivant dans un parfum de scandale, ni même un masque bizarre et ludique... Il est peut-être tout cela à la fois, mais il est d'abord l'image déformée de Witkiewicz dans le regard de l'Autre. Il est Witkiewicz, tel que Witkiewicz se cherche et se perçoit (veut se percevoir?) dans le regard de son public. Avec Witkacy, Witkiewicz met en scène sa propre réception. Witkacy est, pourait-on dire encore, un bouc émissaire que Witkiewicz propose à la communauté des lecteurs afin qu'elle le charge non de tous les maux dont elle souhaite purifier Witkiewicz, mais des fantasmes dont elle-même enveloppe la figure de l'auteur, des fantasmes dont l'hypocrite auteur désire tant se voir enveloppé.

\section{Witkacy, Witkiewicz, et les Polonais}

C'est la relation d'un écrivain à son public qui est ainsi figurée et soumise à la réflexion. Pour en revenir à notre idée initiale, il semble possible de dire que la question du nom cristallise l'essentiel de cette problématique... Revenons donc à nos deux noms: Witkiewicz et Witkacy. Si le premier, Witkiewicz, possède la morphologie typique des noms de famille en polonais, la forme du deuxième, Witkacy, est plus ambiguë: a priori ce n'est pas celle d'un nom de famille, ce pourrait être celle d'un prénom mais ce n'est pas un prénom répertorié, enfin la terminaison en - y est caractéristique des adjectifs. Ajoutons que Witkacy fonctionne toujours en autonomie: jamais aucun prénom, ni un nom ne l'accompagnent. Et si le nom "Witkacy", dont il est convenu de dire qu'il est un pseudonyme, était en fin de compte un surnom, c'est-à-dire une "désignation qu'on substitue au véritable nom d'une personne" ? En effet, force est de reconnaître que "Witkacy" n'est pas à proprement parler un pseudonyme, c'est-à-dire un nom destiné à désigner un aspect de la personne Witkiewicz, mais bien plutôt une manière de désigner la personne Witkiewicz par un groupe précis (en l'occurrence par son public), c'est-à-dire un surnom.

Remarquons en passant qu'on ne donne de surnom qu'à ce qui est familier, proche, investi d'une charge affective. Rappelons d'autre part que l'utilisation de surnoms témoigne d'un sentiment de communauté au sein du groupe, voire en renforce l'unité. Voilà qui nous fait passer de la sphère du littéraire à celle du social. Witkacy est un Witkiewicz "assimilé" par la communauté de ses lecteurs. Le Witkiewicz des Polonais? Car il semble bien que les lecteurs polonais soient les seuls à utiliser obstinément le pseudonyme (ou le surnom). En France, Witkiewicz s'appelle Witkiewicz. Comme si en 
transportant Witkiewicz en terre étrangère, en l'extrayant de sa communauté d'origine, on le débarrassait de Witkacy. Witkacy, pseudonyme devenu surnom, renvoie peut-être même à un traitement typiquement polonais des "grands hommes»: traitement qui connote la province, une familiarité déplacée, une appropriation gênante. Mais, il ne fait nul doute que Witkiewicz ne tire son épingle du jeu. La relation de l'hypocrite auteur à son captif public est pour le moins ambiguë.

L'histoire de Witkiewicz et de Witkacy est celle d'un ménage à trois, comprenant Witkiewicz, Witkacy bien sûr, et l'Autre, le lecteur-usager du nom. En jouant sur les notions de pseudonyme et de surnom, avec Witkacy, Witkiewicz se construit un personnage public, qu'il construit tout en faisant comme s'il était construit par le public lui-même, et tout en feignant de le déconstruire, dans le souci de promouvoir une image soi-disant "vraie" de lui-même, tout en alimentant avec jouissance la fausse. C'est à s'y perdre et à y perdre la figure de l'auteur. Telle est la stratégie witkiewiczienne, qui consiste à programmer sa propre réception en la déstabilisant à l'avance. Le flottement de la nomination dans les discours sur Witkiewicz en atteste-t-il le succès? Une semi-réussite? L'échec? Quelle que soit la réponse à cette question, l'étrange cas de Witkiewicz et Witkacy, le glissement subtil du pseudonyme au surnom qui se produit dans l'œuvre et la vie de Stanislaw Ignacy Witkiewicz, est plus largement symptomatique d'un mode de relations entre un auteur et son public, en d'autres mots de l'ambivalence de la notion d'auteur. 